\title{
Transnationalität und nationale Schulsysteme: Perspektiven für Forschung und Lehre
}

\section{Priska Sieber}

Die Fragen des vorliegenden Themenhefts zu Konsequenzen der Entwicklungen von Schulen in transnationalen Lebenswelten für Forschung und Lehre in der Lehrerinnen- und Lehrerbildung, werden im folgenden Beitrag unter besonderer Berücksichtigung des Deutschschweizer Kontexts bearbeitet. Es wird die These vertreten, dass Forschung, die innovativ ist, vermehrt schulische Prozesse untersucht, Kontexte als nationalstaatlich geprägte Konfigurationen konzeptualisiert sowie mögliche Auswirkungen auf die schulische Praxis mitreflektiert. Für die Lehre wird entsprechend eine differenzierte Auseinandersetzung mit (nationalen) "Normalitäten " in Schulen angeregt und die grosse Bedeutung von praxisorientierten Methoden betont. Zudem werden Hinweise zum Entwicklungsbedarf der Pädagogischen Hochschulen selbst gemacht.

\section{Einleitung}

Die theoretische, empirische und praktische Auseinandersetzung mit Folgen der Migration für Institutionen der Bildung und Erziehung sowie für Individuen im Bildungsprozess ist von verschiedenen disziplinären Traditionen geprägt und hat sich entsprechend in den vergangenen dreissig Jahren stark ausdifferenziert (Gogolin, 2002; Hamburger, 1988). Dies kommt unter anderem in der aktuellen Debatte um den angemessenen Begriff für die Thematik zum Ausdruck. Während auf der einen Seite am Begriff der "Interkulturellen Pädagogik» festgehalten wird, versuchen andere das aus spezifischer Perspektive erschlossene Feld mit einem angepassten Begriff zu erfassen. In der Deutschschweiz tragen beispielsweise die Fachbereiche der Pädagogischen Hochschulen, die sich mit entsprechenden Themen befassen, Namen wie «Interkulturelle Pädagogik», «Migration und Bildung», "Heterogenität», "Schule und Heterogenität», "Umgang mit Vielfalt», "Diversity» oder "Sozialisation und Differenz» (Sieber \& Bischoff, 2007; Vogel, 2006). Jeder dieser Begriffe verbirgt die vielschichtigen Fragen und Zugänge, die sich im Zusammenhang mit demografischen und kulturellen gesell- 
schaftlichen Entwicklungen als Folge der Globalisierung und Individualisierung moderner Gesellschaften für schulische Bildung und Erziehung ergeben. Allen Bezeichnungen mit Begriffen wie Migration, Einwanderung oder Interkulturalität ist im deutschsprachigen Raum zudem gemein, dass sie durch historisch geprägte Konnotationen belastet sind, die in der «Ausländerpädagogik» wurzeln. Assoziationen mit Problemen der «Ausländer» und Familien mit Migrationshintergrund für die allgemeinbildende Volksschule wurden von der Disziplin selbst mitkonstruiert, was noch auszuführen sein wird.

Letztendlich sind jedoch in wissenschaftlichen Debatten terminologische Entscheidungen weniger bedeutsam als die konzeptionellen Grundlagen (Bade \& Bommes, 2004). Erst diese erlauben es, Dimensionen von Bildung und Migration weiter aufzuschlüsseln und aufeinander zu beziehen, um auf dieser Basis eine differenzierte Erfassung etwa der Folgen von transnationalen Lebenswelten auf Schulen und vice versa zu ermöglichen.

Der im vorliegenden Heft im Mittelpunkt stehende Begriff der «Schulen in transnationalen Lebenswelten» verbirgt ebenso vielschichtige Fragen und $\mathrm{Zu}-$ gänge, eröffnet jedoch Chancen für eine konstruktive Anreicherung der Auseinandersetzungen. Der Begriff des Transnationalen beschreibt (1) Vergesellschaftungsformen und soziale Räume, die über nationalstaatliche Grenzen hinweg wirken. In dieser Begrifflichkeit scheint bereits die Konfliktivität mit dem Schulwesen auf, das seit seinem Ursprung eine der zentralen Institutionen des modernen Nationalstaates darstellt ${ }^{1}$. Diese Konfliktivität fruchtbar zu bearbeiten, dürfte wohl als das zentralste Thema gelten. Transnationalität verweist (2) auch darauf, dass Migration keinem linearen, mechanistischen, allgemeingültigen Modell eines unidirektionalen Prozesses von Auswanderung, Einwanderung und Integration (beispielsweise mittels Sozialisation in der Schule) folgt und damit nicht im Verschwinden von Besonderheiten mündet. Vielmehr werden durch Transmigration überall, auch in den Schulen, neue kulturelle und soziale Konstellationen erzeugt. Und schliesslich beziehen sich (3) die Diskurse, in denen der Begriff des Transnationalen bis anhin mehrheitlich verwendet wurde, tendenziell auf sozial respektierte Formen von Migration, sei dies beispielsweise im Zusammenhang mit transnationalen Unternehmen oder bezogen auf die Hochschulbildung. Damit wird sowohl die Ungleichheitsthematik angesprochen als auch der Problematisierung von Migration eine ressourcenorientierte Perspektive angefügt. Der aus der Verstehenden Soziologie stammende Begriff der Lebenswelten $^{2}$ verweist auf die alltäglichen, je subjektiven Wirklichkeitsregionen, die beispielsweise transnational strukturiert sein können. Diese Lebenswelten stellen die Struktur des Bewusstseins und den praktischen Vorrat des Wissens dar, die im Laufe der Sozialisation erworben werden und die aktive Teilnahme am Alltag beispielsweise in der Schule oder in transnationalen sozialen Räumen ermöglicht. Im Lebensweltkonzept kann der Mensch aber auch in seine Lebenswelt eingreifen und sie verändern. «Zugleich beschränken die in diesem Bereich vorfindlichen Gegenständlichkeiten und Ereignisse, einschliesslich des Han- 
delns und der Handlungsergebnisse anderer Menschen, seine freien Handlungsmöglichkeiten. Sie setzen ihm zu überwindende Widerstände wie auch unüberwindbare Schranken entgegen» (Schütz \& Luckmann, 1975, S. 23).

Damit verweist der Begriff der "Schulen in transnationalen Lebenswelten» auf zentrale Themen und Fragestellungen, die neue Ansätze in Forschung und Lehre aufgreifen sollten und diese befruchten dürften: Wie bearbeiten nationale Bildungssysteme die Konfliktivität mit transnationalen Vergesellschaftungsformen? Welche neuen Konstellationen entstehen in Schulen durch die vielfältigen Orientierungen und die Unabgeschlossenheit von Integrationsprozessen? Wie bewerten die schulischen Akteure Transmigrationsprozesse unterschiedlicher Bevölkerungsgruppen bzw. auf unterschiedlichen Ebenen des Systems? Und schliesslich, wie bearbeiten sie die historisch tief verwurzelten, an Nation orientierten strukturellen Schranken des Systems (z.B. die Gruppierung der Schülerschaft, Curricula, die Bewertungsnormen oder intersubjektiven Theorien zum Lernen der Schülerinnen und Schüler)?

\section{Kontextualisierte Forschung}

\section{Lücken überwinden}

Im deutschsprachigen Raum hat wohl kein anderer Zugang die Auseinandersetzung mit Migration und deren Folgen für die Schule so stark geprägt wie die bildungssoziologische Ungleichheitsforschung. Seit über zwanzig Jahren - also seit den Anfängen der empirischen Auseinandersetzung mit den entsprechenden Fragen - untersucht sie das Phänomen ungleicher Bildungschancen von Kindern und Jugendlichen mit verschiedenem sprachlichen, ethnischen und soziokulturellen Hintergrund. Dabei wird immer wieder festgestellt, dass Kinder und Jugendliche mit Migrationshintergrund in Sonderklassen sowie in Sekundarschulen mit tiefen Leistungsanforderungen übervertreten sind, sich die Situation über die Zeit sogar verschlechtert hat (Kronig, Haeberlin \& Eckhart, 2000; Lischer, 1997; SKBF, 2006) und dass sich die Benachteiligung beim Übergang in die Berufswelt (Haeberlin, Imdorf \& Kronig, 2004) und in weiterführende Schulen fortsetzt. Der Erklärungswert bezüglich der Ursachen des Phänomens ist seitens der bildungssoziologischen Ungleichheitsforschung allerdings gering. Andere wissenschaftliche Perspektiven haben dazu im Laufe der vergangenen Jahre zusätzliche Erklärungsansätze ergründet (Becker \& Lauterbach, 2007). Verkürzt formuliert gelten je nach verwendeter theoretischer Perspektive als Gründe für die Benachteiligung von Kindern und Jugendlichen mit Migrationshintergrund etwa

- deren sprachliche Defizite oder fehlende kulturelle und materielle Ressourcen in der familiären Sozialisation (ätiologische Ansätze);

- ignorierende und stigmatisierende Interaktionen zwischen Lehrpersonen und gewissen Schülerinnen und Schülern sowie unzureichende Förderung (bei- 
spielsweise des Zweitsprachenerwerbs) als Folge lückenhafter Kompetenzen von Lehrpersonen (begegnungsorientierte Ansätze);

- die schulischen Strukturen, in denen eine enge Normalitätsvorstellung gekoppelt an frühe Selektion institutionalisiert ist (institutionelle Ansätze). Alle diese Ansätze erklären einen Teil des Problems, doch allen Ansätzen ist etwas gemein: Sie vermögen einerseits die grossen Unterschiede zwischen verschiedenen Schulsystemen (beispielsweise in der Schweiz) sowie innerhalb von Schulsystemen nur unzureichend zu erklären und sie bieten kaum Grundlagen dafür, warum sich verschiedene Migrantengruppen (z.B. griechische, türkische oder portugiesische) statistisch signifikant unterscheiden und die Unterschiede innerhalb dieser Gruppen ebenfalls beträchtlich sind (wobei erfolgreiche Beispiele nur selten untersucht wurden).

Im weiteren kann der bisherigen wissenschaftlichen Auseinandersetzung mit dem Thema vorgeworfen werden, dass sie in der Praxis kaum eine Wirkung gezeigt hat und sich im Schulalltag vor allem ätiologische Erklärungsmuster als Ursachen für Schulmisserfolg von Kindern und Jugendlichen mit Migrationshintergrund niedergeschlagen haben, mit denen eine Pathologisierung der Lebenspraxis von Migrantinnen und Migranten einhergeht.

Innovative Forschung müsste insbesondere diese Mängel überwinden und dazu unter anderem Folgendes berücksichtigen:

- Forschung sollte weniger nach Einzelfaktoren für Benachteiligung oder anderen Migrationsfolgen für Individuum und Schule suchen, denn diese sind immer multifaktoriell bedingt. Entsprechende Untersuchungen bergen die Gefahr der Variablenkonfundierung und sind nur selten in der Lage, genaue Auskunft über die Art und Weise des Einflusses zu geben. Zudem bleiben biund multidirektionale Geschehnisse, wie sie insbesondere in der LehrerSchüler-Interaktion oft vorkommen, unberücksichtigt, und oft werden die wirkungsvollsten Faktoren im Schulumfeld lokalisiert. Innovative Forschung sollte vielmehr die Komplexität der Mechanismen rekonstruieren, Benachteiligung und Bevorzugung als Konfiguration von Faktoren verstehen und die Prozesse bestimmen, die zu bestimmten Migrationsfolgen für Schulsysteme und Individuen in schulischen Lernprozessen führen. Es braucht also vermehrt Untersuchungen, die institutionelle Praktiken, programmatische Selbstfestlegungen, organisatorische Konventionen, implizite Regeln und eingespielte Routinen offen legen (Radtke, 2004).

- Forschung sollte weniger zeitlich und räumlich isolierte Phänomene untersuchen, sondern vergangene und gegenwärtige gesellschaftliche Transformationsprozesse berücksichtigen, die sich in je unterschiedlichen sozialen und kulturellen Kontexten mit den jeweiligen pluralen Formen von Handlungsorientierungen und Strukturen verschieden auswirken. Es braucht vermehrt Untersuchungen, die Transnationalität und deren Folgen für die Schulen in einem beschreibbaren Gesamtkontext als spezifische Konfiguration untersuchen. 
- Forschung sollte zudem ihre mangelhafte oder gar negative Wirkung in der Schulpraxis überwinden, indem die Auswirkungen der Forschung bereits in der Konzeptionsphase mitreflektiert werden. Dazu gehören etwa Überlegungen, wie die Forschungsergebnisse verwendet und angewendet werden sollen und wie allenfalls die Erforschten und/oder Nutzenden in den Forschungsprozess einzubeziehen sind.

In den letzten Jahren sind diesbezüglich interessante erste Untersuchungen vorgelegt worden, auf die in den folgenden Ausführungen selektiv und punktuell eingegangen wird.

\section{Konzeptionelle Zugänge und thematische Akzente}

Relevante Themen und Fragestellungen einer Forschung, die innovativen Charakter bezüglich eines noch jungen und entsprechend unzureichend erforschten Bereichs wie demjenigen von Schulen in transnationalen Lebenswelten aufweisen, gibt es viele. In der Einleitung dieses Beitrags wurden einige davon angesprochen. Sie sind vielfältig und betreffen etwa verschiedene Ebenen des Schulsystems, einschliesslich deren Kontexte oder unterschiedliche Akteurgruppen in je spezifischen (transnationalen) Lebenswelten. Unzureichend erforscht sind etwa - die Wirkung unterschiedlicher Modelle des Mehrspracherwerbs auf Schulen und deren Kontexte bei mehrfacher räumlicher und sprachlicher Orientierung einer zunehmenden Anzahl von Schülerinnen und Schülern,

- das Ausmass und die erwünschten sowie unterwünschten Ergebnisse der Umsetzung verschiedener Zugänge von interkulturellem Lernen im Schulalltag,

- die Folgen der aus dem angelsächsischen Raum stammenden Standard-Diskussionen auf soziales Lernen in transnationalen Lebenswelten oder

- die interkulturellen Kompetenzen des (Hoch-)Schulpersonals, was diese genau umfassen und wie sie effektiver erworben werden könnten.

Als besonders innovativ - so die These hier - ist Schulforschung, die Mechanismen und Prozesse vor dem Hintergrund eines spezifischen, historisch entstandenen Kontextes untersucht sowie mögliche Auswirkungen auf konkrete Praxis mitreflektiert: Innovative Schulforschung fängt das vielschichtige Verhältnis zwischen Akteuren und System bzw. zwischen Handlung und jeweiliger Struktur ein, ganz im Sinne des Lebensweltkonzepts, und macht die Ergebnisse nutzbar für eine konstruktive Weiterentwicklung der schulischen Praxis in transnationalen Kontexten.

Für alle Forschungsvorhaben geht es auf einer allgemeinen Ebene darum, zu verstehen, wie nationale Schulsysteme ${ }^{3}$ in der Vergangenheit gestaltet wurden, welche und wessen Vorstellungen sich in einem spezifischen System durchsetzen konnten (und welche nicht) und heute in Form von institutionellen Strukturen in Gesetzestexten, Reglementen, Verordnungen, Curricula und Lehrbüchern der bürokratischen Organisation Schule festgeschrieben sind. Diese institutionellen Strukturen beschreiben zu einem beachtlichen Teil diejenige schulische Lebenswelt, die die freien Handlungsmöglichkeiten beschränkt, überwindbare Wider- 
stände oder unüberwindbare Schranken setzt (Schütz \& Luckmann, 1975). Einige entsprechende Studien, die Gestaltungsdiskurse in der Vergangenheit analysieren und damit einen wichtigen Beitrag zum Verständnis der nationalstaatlichen Gestalt von Schulsystemen leisten, liegen bereits vor. Sie beschreiben beispielsweise die historische Genese des sprachlichen Selbstverständnisses der heutigen deutschen Schulen ADDIN(Gogolin \& Nauck, 2000) und wie sich kulturelle Selbstverständlichkeiten und Weltsichten hinter der Oberfläche der schulischen Inhalte verbergen (Fend, 2006, S. 58ff.) oder wie die historisch frühe und ausgesprochen gründliche Institutionalisierung einer heilpädagogischen Domäne in den Schulsystemen der Schweiz eine Logik der Separierung etablierte, die gleichzeitig die Normalitätsvorstellung in Regelklassen verengte (Sieber, 2006; 2007). Solche Studien ermöglichen, dass die Geschichte der Entstehung eines Gefüges von (nationalen) Grundüberzeugungen und Praxen nicht als Geschichte in Vergessenheit geraten (Gogolin, 1994) und als soziale Konstruktionen entlarvt werden, die Machtverhältnisse definieren und die Handlungsmöglichkeiten der schulischen Akteure beschränken. Nur wer die Gestalt und Gestaltung von nationalen Schulsystemen versteht, kann Widerstände in der aktuellen Schulpraxis bezüglich transnationaler Lebenswelten reflektieren und allenfalls überwinden sowie unüberwindbare Schranken in künftigen Schulreformbestrebungen fundiert bearbeitbar machen (Sieber, 2006).

Vor diesem Hintergrund sollte Forschung die Praktiken in konkreten schulischen Kontexten in unterschiedlichen transnationalen Lebenswelten untersuchen. Die in historisch gestalteten nationalen Schulsystemen institutionalisierten Strukturen stellen nämlich ein komplexes Regelwerk zur Anleitung von Handlungen dar, das nicht nur das schulische Personal durch seine lebensweltlichen Erfahrungen internalisiert hat, sondern auch die über längere Zeit damit konfrontieren Schülerinnen und Schüler, Eltern, Politikerinnen oder Lehrerbildner. Die institutionellen Vorgaben erfahren allerdings Modifikationen, die sich einerseits aus dem spezifischen lokalen Kontext ergeben und anderseits durch die generell subjektive Verfasstheit der Handelnden bewirkt werden. Die MakroVorgaben der nationalen Schulsysteme erfahren also auf der Mikroebene eine situationsspezifische, allenfalls auf transnationale Lebenswelten angepasste Transformation, sodass erst im konkreten Handeln und Aushandeln im Schulalltag beispielsweise Schulerfolg oder -misserfolg, Sonderklassenzuweisungen, Anerkennung, Ablehnung, Zugehörigkeit oder «natio-ethno-kulturelle Zugehörigkeit» ${ }^{4}$ (Mecheril, 2003, S. 118ff.) definiert wird. Forschung, die in kontextualisierten, strukturell informierten, bereichsspezifischen, detaillierten empirischen Fallstudien solche Definitionsprozesse rekonstruiert, sie als Produkt von Handeln in Interaktionen konzeptualisert, kann wichtige neue Erkenntnisse liefern, etwa darüber, welches Wissen in institutionalisierten Prozessen im Schulalltag für das Handeln leitend ist (und welches nicht), aus welchen Wissensbeständen das Wissen stammt (Institution, Profession und Politik), woran sich die Akteure bei ihrer Handlungswahl orientieren (und woran nicht), wie sie institutionalisiertes 
Wissen aufgreifen, umsetzen, nutzen und anpassen. So können fundierte Antworten etwa darauf gefunden werden, in welchen Situationen und wozu Handelnde ethnische Unterscheidungen benützen oder ignorieren, wie Zugehörigkeit oder Ausschluss definiert wird, welche Mechanismen bei positiven oder negativen Selektionsentscheiden wirksam werden oder wie schulische Akteure auf Wandel im transnationalen Kontext der Schule reagieren. Solche Studien haben etwa Cicourel und Kitsuse (1974), Struck (2001) sowie Gomolla und Radtke (2002) vorgelegt und Übergänge innerhalb des Bildungssystems rekonstruiert. Interessante Erkenntnisse unter anderem über positiv gestaltete Integrationsprozesse in verschiedenen Schulen liefern die Fallstudien von Sieber (2006). Wertvolle Einsichten bietet zudem die ethnografische Schulforschung, wenn diese strukturell informiert angegangen wird, wie beispielsweise das Forschungsprojekt zu «Schulen in der transnationalen Gesellschaft», das untersuchte, mit welchen Strategien schulische Akteure im Hinblick auf die Aufrechterhaltung schulischer Funktionalitäten dem Leistungs- und Wettbewerbsdruck im schulischen Umfeld begegnen (Fiechter \& Kappus, 2006; Oester, Fiechter \& Kappus, 2005).

Die oben angedeuteten konzeptionellen Zugänge und thematischen Akzente können auch dem dritten hier formulierten Anspruch an innovative Forschung gerecht werden. Wenn Forschung die wechselseitige Beziehung zwischen (nationaler) Normierung und (transnationaler) Realität im Schulsystem herauszuarbeiten vermag, leistet sie ein Mehrfaches, das auch für die Praxis von hoher Relevanz ist: (1) Sie macht die Wirksamkeit verschiedener Aspekte des offiziellen Schulprogramms bewusst; (2) Sie verdeutlicht die Eigenlogik bzw. Selbstreferenzialität der Umsetzung der Systemvorgaben und macht damit auf die Veränderbarkeit aber auch auf Widerstände schulischer Lebenswelt aufmerksam; (3) Sie macht Probleme sichtbar, an denen sich Akteure in transnationalen Kontexten abarbeiten; (4) Sie verweist auf unüberwindbare Schranken und betont damit die Notwendigkeiten von Programmneuformulierungen bezüglich unterschiedlicher Handlungsbedingungen in den Schulen. Mittels der Erforschung von erfolgreichen Schülerinnen und Schülern sowie der Schulen und Systeme, die dazu beigetragen haben, können zudem strukturelle und organisatorische Veränderungspotenziale und wirksame pädagogischen Ansätze für die Praxis erarbeitet werden.

\section{Methoden und zu beteiligende Disziplinen}

Praxisrelevanz entsteht zudem bei der Darstellung der Forschungsergebnisse. Nur wenn die Bildungsforschung ihre Erkenntnisse in einer Art und Weise präsentiert, die die verschiedenen Zielgruppen wie Lehrpersonen, Eltern, Schulleitungen, Bildungsverwaltung, Bildungspolitik und breite Öffentlichkeit wahrnehmen und verstehen, kann sie Schulrealitäten beeinflussen.

Die Zugriffsweise der Forschung auf die oben skizzierten Gegenstände bewegt sich weitgehend im Rahmen der gebräuchlichen Methoden historischer Forschung und empirischer Sozialforschung, wobei insbesondere vergleichenden $\mathrm{Zu}$ - 
gängen eine wichtige Bedeutung zukommt. «Besonderheiten» können nur im Vergleicht mit "Normalem» bzw. «Erwünschtem» festgestellt werden. Dem ist insbesondere bei der Wahl der Fälle Rechnung zu tragen, sei dies bei der Wahl von nationalen Schulsystemen, deren historische Gestalt analysiert werden soll, oder bei der Wahl von Schulen und Akteurgruppen, deren Handeln in Interaktionen im Hinblick beispielsweise auf das Schaffen von Zugehörigkeiten oder die Gestaltung von Lernprozessen untersucht wird. Dabei ist die Untersuchung von "erfolgreichen» Fällen besonders fruchtbar, weil sie erhellen können, wie und in welchen Kontexten die in nationalen Schulsystemen institutionalisierten Widerstände und Schranken überwunden werden können. Neue Erkenntnisse erzielten etwa systematische Vergleiche von Fällen, in denen Transnationalität positiv und negativ bewertet wird, also beispielsweise ein Vergleich der Prozesse in Schulen in stark und schwach transnationalen Kontexten, ein Vergleich der Diskurse auf Hochschulebene und Volksschulebene oder ein Vergleich der Prozesse in den aktuell wie Pilze aus dem Boden schiessenden internationalen Primar- und Sekundarschulen und den öffentlichen Volksschulen.

Um die komplexen Mechanismen, die mehrschichtigen institutionellen Kontexte, verschiedene individuelle Akteure und Akteurgruppen in ihren jeweiligen Handlungsorientierungen, Wahrnehmungen und interaktiven Beziehungen gleichermassen systematisch und fundiert erfassen zu können, sind Forschungsdesigns notwendig, die kaum von individuellen Forschenden stringent konzeptualisiert und methodisch sauber umgesetzt werden können. Multidisziplinäre Teams mit interdisziplinärer Forschungserfahrung sind dafür vorzusehen, deren Zusammenarbeit allenfalls in neue transdisziplinäre Ansätze mündet.

\section{Institutionelle und organisatorische Veränderungen}

Um relevante, konzeptionell und methodisch stringente Schulforschung in der oben skizzierten Komplexität betreiben zu können, braucht es nicht nur erfahrene und motivierte Forscherinnen und Forscher, die über Projektmanagementkompetenzen verfügen und gut mit den jeweiligen Forschungsgemeinschaften sowie mit dem schulischen und bildungspolitischen Feld vernetzt sind. Nötig sind dafür insbesondere institutionelle Rahmenbedingungen die helfen, solche Kapazitäten aufzubauen, sie zu entfalten und zu halten.

Diesbezüglich bestehen in der Schweiz und speziell in der Deutschschweiz prekäre Verhältnisse, nicht nur was die «interkulturelle Bildungsforschung» 5 angeht, sondern bezüglich der Bildungsforschung allgemein, obwohl Bildung grundsätzlich als wichtiger gesellschaftlicher Bereich anerkannt wird. Seit den 1960 er-Jahren wird in der Schweiz von unterschiedlichen Instanzen und in verschiedenen Berichten immer wieder die Rückständigkeit der Bildungsforschung in der Schweiz moniert (Gretler, 1994; Hofstetter \& Schneuwly, 2001; OECD/CERI, 2007). Das Credo bleibt seit Jahrzehnten das Gleiche: Die Bildungsforschung ist fragmentiert, kaum international oder mit anderen Disziplinen vernetzt, findet in heterogenen institutionellen Strukturen statt, zeigt nur 
geringe Wirkung auf Politik und Verwaltung, erhält im Vergleich mit anderen OECD-Ländern unterdurchschnittliche Ressourcen, hat keine kritische Masse, sodass (junge) Bildungsforscherinnen und -forscher in der Schweiz weitgehend isoliert sind. ${ }^{6}$ Eine aktuelle Untersuchung zur «interkulturellen Bildungsforschung» in der Schweiz zeigt entsprechend, dass trotz Zunahme der Forschungstätigkeiten in der Deutschschweiz in den Jahren 1993-2006 nur gerade 41 Forschungsprojekte durchgeführt wurden, die Grosszahl davon Qualifikationsarbeiten oder Auftragsprojekte (Gakuba, Gremion, Hutter, Ogay \& Zharkova, i.V.). Entsprechend ist Bildungsforschung in der Deutschschweiz in der Regel eine «biografische Episode» (Criblez, 1998, S. 181) und interkulturelle Bildungsforschung ein seltenes Ereignis, weil es keine Universitäten gibt, an denen entsprechende Inhalte angesiedelt sind.

Die Gründe für die Marginalisierung, Fragmentierung und Isolierung liegen insbesondere im föderalen Bildungssystem der Schweiz, in dem auch Bildungsforschung massgeblich aufkantonaler Ebene gesteuert und finanziert wird und keine zentrale Institution eine Forschungsstrategie festlegt. Ob die jüngst in der Schweiz umgesetzte Tertiarisierung der Lehrerinnen- und Lehrerbildung, mit der unter anderem das Ziel verfolgt wird, die lehrberufsspezifische Forschung und Entwicklung zu stärken (EDK, 1995), etwas daran ändern wird, bleibt abzuwarten. Die 15 Pädagogischen Hochschulen, die seit 2001 ihren Betrieb aufgenommen haben, haben mittlerweile in national kaum gesteuerten Prozessen eigene Forschungsdepartemente entwickelt. An einigen scheint sich ein Schwerpunkt im Bereich "Migration und Bildung" herauszukristallisieren (Vogel, 2006) ${ }^{7}$. Dadurch haben sich zwar die Ressourcen für Bildungsforschung etwas erhöht, doch die kantonale Steuerung und Finanzierung bleibt unangetastet, sodass auch künftig die Gefahr von Fragmentierung und Isolierung bestehen bleibt.

«Interkulturelle Bildungsforschung» bräuchte demgegenüber koordinierte Forschungsinstitutionen, in denen ausreichend Ressourcen zur Verfügung stehen, um entsprechende Forschungskapazitäten aufzubauen, zu entfalten und zu halten, sodass sich kompetente Forschende mit den jeweiligen nationalen und internationalen Forschungsgemeinschaften sowie mit dem schulischen und bildungspolitischen Feld vernetzen können. Dies würde das Lernen im System deutlich verbessern und könnte insbesondere die Wirksamkeit der Forschung erhöhen.

\section{Lehre zur Förderung einer reflektierten Praxis}

\section{Lücken und aktuelle Umsetzung}

In der Literatur wird der "Interkulturellen Pädagogik» 8 in der Aus- und Weiterbildung von Lehrpersonen tendenziell - nicht nur für die Schweiz - eine marginale Bedeutung attestiert (Allemann-Ghionda, de Goumoëns \& Perregaux, 1999; 
Cochran-Smith, Davis \& Fries, 2004; Krüger-Potratz, 2003). Die Dimension der kulturellen und sprachlichen Pluralität der Schülerschaft sei kaum Thema der Lehrerinnen- und Lehrerbildung, werde allenfalls in Einzelkursen, Wahlpflichtangeboten oder Zusatzstudiengängen thematisiert und dann vorwiegend auf die Erörterung der (negativen!) Auswirkungen der Migration beschränkt. Nur an wenigen Institutionen werde "Interkulturelle Pädagogik» weitergehend betrieben, oft aufgrund der Initiative einzelner Dozierender, jedoch ohne dass die Disziplin ein offizieller Lernbereich wäre und oft ohne die Dimension des Zweitspracherwerbs zu thematisieren.

Die Gründe für die Marginalisierung des Themas sind zahlreich. Dazu gehört insbesondere, dass «Interkulturelle Pädagogik» eine relativ junge Domäne ist, wenig Personal zählt und die Implementierung von Neuem in bestehende bürokratische Strukturen generell ein komplexer politischer Prozess ist und entsprechend viel Zeit benötigt. Dazu gehören aber auch Schwierigkeiten, die in der Domäne selbst begründet sind. Erstens hat sich die «Interkulturelle Pädagogik» stark ausdifferenziert, und es besteht allgemein Uneinigkeit darüber, was die zentralen Kompetenzen sind, die Lehrpersonen in der Lehrinnen- und Lehrerbildung erwerben sollten. Aktuell vorliegende Kompetenzkataloge sind umfangreich (EDK/CDIP, 2000) und bedürfen, um umsetzbar zu sein, einerseits der Unterscheidung nach Kompetenzen, die von allen Lehrpersonen in der Grundausbildung zu erwerben wären, und Kompetenzen, die in einer Spezial- oder Zusatzausbildung erworben werden könnten, sowie anderseits der Integration eines Grossteils der Inhalte in bestehende Fachcurricula. Zweitens weist die Forschung bezüglich Fragen der entsprechenden Kompetenzentwicklung, von denen viele den affektiven, behavioralen, kognitiven und reflexiven Bereich gleichermassen betreffen, auf eine hohe Komplexität der Lernprozesse hin, die zu organisieren keine einfachen Lösungen zulässt.

In der Schweiz hat sich durch den unlängst implementierten Umbau der Lehrerinnen- und Lehrerbildung eine Chance eröffnet, die "Interkulturelle Pädagogik» in der Lehre neu zu verorten. Unterstützt wurde dies durch den grundsätzlich dafür vorhandenen politischen Willen. In den nationalen Reglementen zur Anerkennung der neuen Lehrdiplome ist verbrieft, dass der Bereich der Erziehungswissenschaften Aspekte der Interkulturellen Pädagogik umfassen muss (EDK, 1999a, 1999b). Dazu wurden zudem umfassende Empfehlungen formuliert (EDK/CDIP, 2000). Eine neue Untersuchung zur Umsetzung dieser Empfehlungen an den noch jungen Pädagogischen Hochschulen der Schweiz zeigt denn auch, dass sich die Situation deutlich verändert hat (Sieber \& Bischoff, 2007). An 11 von 16 antwortenden Institutionen ${ }^{9}$ wurden Personen als Verantwortliche für «Interkulturelle Pädagogik» bezeichnet und mit Ausnahme von zwei Institutionen ist überall mindestens ein obligatorisches Modul mit entsprechenden Inhalten in das Curriculum aufgenommen worden. An einzelnen Hochschulen können Studierende über ein Dutzend Module zu entsprechenden Fragen belegen. 
Im Rahmen der genannten Untersuchung wurden die befragten Fachleute der Pädagogischen Hochschulen gebeten, Konzepte, Module, Inhalte, Regelungen oder Praktiken im Bereich «Interkulturelle Pädagogik» an ihren Hochschulen anzugeben, die sie persönlich als besonders innovativ, effektiv oder aussergewöhnlich beurteilen würden. Die Befragten nannten insgesamt zwanzig Beispiele. Als «Gute Praxisbeispiele» der «Interkulturellen Pädagogik» wurden genannt (COHEP, 2007): Gesamtkonzepte der Lehrerinnen- und Lehrerbildung (3 Beispiele), Nord-Süd-Austauschprogramme (3), Studienwochen (2), Auslandpraktika (2), ein Mobilitätskonzept (1), Zusammenarbeitsformen mit Fachstellen (1), Unterrichtsmethoden wie Fallbearbeitungen, «problem-based learning», Sensibilisierung, sozialanthropologische Methode oder globales Lernen im Praktikum (5), Module der politischen Bildung (1), ein Nachdiplomkurs in Deutsch als Zweitsprache (1) und ein Forschungsprojekt zur Kultur der eigenen Pädagogischen Hochschule (1). Dies verweist nicht nur auf neue, innovative Ansätze in der Lehre, sondern auch auf die vielfältigen Anforderungen an «Interkulturelle Pädagogik».

\section{Themenschwerpunkte}

Die Frage nach Themenschwerpunkten, die sich die Lehre der Lehrerinnen- und Lehrerbildung bezüglich einer Schule in transnationalen Lebenswelten setzen sollte, ist zunächst einfach zu beantworten: Es geht um die Auseinandersetzung mit "Normalitäten»: (1) eigenen und denjenigen von anderen; (2) deren Erwerb und Wirkung; (3) auf der Ebene von Gesellschaften, Schulsystemen, Schulen, Unterricht und Individuum. Das klingt einfach, ist allerdings in der Praxis kein leichtes Unterfangen, weil dadurch über weite Strecken diejenigen lebensweltlichen Routinen befragt werden, die die Identitäten der Lernenden betreffen und die das Leben massgeblich vereinfachen. In einem Kontext allerdings, in dem sich lebensweltliche Erfahrungshorizonte zunehmend multiplizieren, führt nur die Fähigkeit zur Relativierung der eigenen «Normalitäten» dazu, Zugehörigkeiten zu ermöglichen. Das Endziel einer Lehrerinnen- und Lehrerbildung müsste entsprechend sein, dass Lehrpersonen in ihren Tätigkeiten nicht «Normalitäten» und Gleichheit von Kultur, Ethnie, Nationalität oder Sprache, sondern Differenz als essentiell und wertvoll erleben - ein hehres Ziel in einem nationalen Schulsystem.

Das setzt ein differenziertes Wissen voraus über Identitätsentwicklungen in verschiedenen Lebenswelten sowie über soziale Konstruktionen von sozialen Differenzlinien in der Gesellschaft und auf verschiedenen Ebenen des nationalen Schulsystems, das es in der Lehre zu thematisieren gilt. Die Lehrerbildung sollte zudem in Methoden einführen, mit denen enge «Normalitäten» differenziert werden können, beispielsweise bezüglich der schulischen Beurteilung von Kindern und Jugendlichen, der methodischen Gestaltung von Lernumgebungen, der inhaltlichen Ausrichtung von Unterricht oder der kommunikativen Möglichkeiten in Interaktionen etwa mit verschiedenen Schülerinnen und Schülern, aber auch mit deren Eltern. 
Mit anderen Worten: In der Lehre im Hinblick auf Schulen in transnationalen Lebenswelten sollte es zentral um Themen gehen, anhand derer die Studierenden eine «Normalität» reflektierende (Schul-)Praxis kennen lernen können.

\section{Methoden}

Will die Lehre allerdings die geschilderte reflektierte Schulpraxis fördern, ist weit mehr als die gewählten Themen zu berücksichtigen. Von zentraler Bedeutung sind die gewählten Methoden (Brown, 2004), will die Lehre bestehende Vorstellungen und Haltungen und letztendlich Identitäten beeinflussen, die in der künftigen Praxis der Lehrpersonen entgegen der aktuell nationalstaatlichen «Normalität» vieler Schulen ihren Niederschlag finden. Dazu ist weit mehr als der Erwerb von Wissen und die Auseinandersetzung mit Texten notwendig. Im Kern geht es darum, Deutungs-, Reflexions- und Handlungskompetenzen insbesondere bezüglich bestehender (nationaler) Orientierungen einzuüben, womit das in der Lehrerinnen- und Lehrerbildung vielfach diskutierte Verhältnis von Theorie und Praxis angesprochen ist.

Aufgrund der aktuellen Forschungsliteratur zur Lehrerinnen- und Lehrerbildung (Übersichten bieten etwa Bennett, 2001; Cochran-Smith et al., 2004; Sleeter, 2001) zeigt sich, dass die genannten Kompetenzentwicklungen nur durch die enge Verzahnung von theoretischer Reflexion und praktischen Erfahrungen gelingen kann, der Verbindung also von affektiven, kognitiven, reflexiven und behavioralen Lernprozessen, wobei auch in der Lehrerinnen- und Lehrerbildung die individuellen, mehrheitlich nationalen statt transnationalen Orientierungen und Erfahrungen der Lernenden einen entscheidenden Einfluss haben und berücksichtigt werden müssen.

Als besonders wirksam erweisen sich forschende Zugänge, eigene reflektierte Arbeitserfahrungen in multikulturellen Klassen und in "fremd-kulturellen" Gemeinschaften bzw. Kontexten. An einigen der neuen Pädagogischen Hochschulen der Schweiz werden bereits viel versprechende Ansätze umgesetzt. Dazu gehören etwa schulische Praktika in Herkunftsländern von Migrantinnen und Migranten, mehrere Monate dauernde Begleitungen eines neu zugezogenen Kindes, Einsätze in Bildungsprojekten der Entwicklungszusammenarbeit oder studentische Forschungsprojekte zur Migrationsthematik, um nur einige zu nennen.

Damit wird deutlich, dass die Konsequenzen von transnationalen Lebenswelten nicht einfach die Lehre in einzelnen Modulen, Fächern oder Disziplinen betrifft, sondern die Lehrerinnen- und Lehrerbildung als Ganzes.

\section{Zu beteiligende Disziplinen im Lehrkörper}

Es stellt sich somit weniger die Frage, welche Disziplinen im Lehrkörper der Lehrerinnen- und Lehrerbildung zu beteiligen sind, als vielmehr die Herausforderung, wie die sprachliche und kulturelle Bewusstheit des gesamten Personals der Hochschulen gefördert werden kann, sodass alle Veranstaltungen in der Lehrerinnen- und Lehrerbildung stärker auf eine Schule in transnationalen Lebenswelten 
vorbereiten. Das Thema ist nicht nur für Dozierende der Erziehungswissenschaften von Bedeutung, sondern betrifft die gesamte fachliche, fachdidaktische und praktische Ausbildung, deren Inhalte und Methoden unter der Perspektive von Transnationalität bzw. Heterogenität (neu) zu konzipieren sind. Dazu gehören insbesondere die Mehrperspektivität der Lehre, aber auch die Auseinandersetzung mit Fragen des Zweitspracherwerbs und die Bedeutung der sprachlichen Bildung für jeglichen Fachunterricht in transnationalen Lebenswelten.

\section{Institutionelle und organisatorische Veränderungen}

In der Schweiz ergab sich die bereits erwähnte glückliche Koinzidenz, dass die Bestrebungen zur Implementierung von «Interkultureller Pädagogik» mit dem Gesamtumbau der Lehrerinnen- und Lehrerbildung zusammenfiel. Dadurch wurde einerseits ein hoher Gestaltungsspielraum eröffnet und anderseits waren die sonst üblichen selbstreferenziellen Besitzstandskämpfe um Inhalte und Umfang abgeschwächt. Entsprechend wurden an der Grosszahl der neuen Pädagogischen Hochschulen Ausbildungsziele formuliert (in der Regel in Form von Standards und Kompetenzen), die unter anderem auf eine Schule in transnationalen Lebenswelten ausgerichtet sind (Sieber \& Bischoff, 2007). Allerdings bricht sich die Umsetzung allzuoft an den Kompetenzen der beteiligten Dozierenden und beigezogenen Praxislehrpersonen ${ }^{10}$, die den monolingualen und monokulturellen Habitus der nationalen Schulsysteme unreflektiert internalisiert haben.

Die grosse institutionelle Herausforderung ist es denn auch, das Hochschulpersonal auf eine Schule in transnationalen Lebenswelten vorzubereiten und in der Hochschulbildung selbst eine Kultur der Anerkennung von Differenz zu institutionalisieren. Dazu eignen sich beispielsweise hochschulinterne Schulentwicklungsprozesse in Kombination mit einer nationalen und internationalen Öffnung der Hochschulen. Dadurch können Prozesse ausgelöst werden, die eine reflexive Praxis bezüglich der teilweise noch engen Normalitätsvorstellungen der Hochschulen begünstigen.

\section{Anmerkungen}

1 Die modernen Pflichtschulen sollten «aus der Sicht ihrer staatlichen Veranstalter und Verwalter die innere kulturelle Einheit der Gemeinschaft konsolidieren, die soziale Über- und Unterordnung reproduzieren und legitimieren, zugleich aber Kohärenz über soziale Standes- oder Klassendifferenzen hinweg befestigen, Loyalität gegenüber dem Staat und seiner Regierung(-sform) erzeugen und nationale Mobilisierung gegen äussere Bedrohung erleichtern» (Radtke, 2004, S. 630).

2 Assoziationen mit EDMUND HUSSERL (1859-1938) und ALFRED SCHÜTZ (18991959), aber auch mit JÜRGEN HABERMAS (*1929) liegen nahe.

3 Auch wenn in den folgenden Ausführungen oft auf die kantonalen Schulsysteme der Schweiz Bezug genommen wird, wird aus konzeptionellen Gründen dennoch der Begriff der «nationalen Schulsysteme» verwendet.

4 Diese Form der Zugehörigkeit wird von Mechril (2003) als zentraler Bezugspunkt einer Pädagogik der Migrationsgesellschaft umschrieben. 
5 Im Folgenden steht der Begriff «interkulturelle Bildungsforschung» für jene Bildungsforschung, die sich im Rahmen der in der Einleitung angesprochenen Konzepte abwickelt.

6 Die Universität Genf in der französischen Schweiz stellt diesbezüglich eine Ausnahme dar. Mit mehr als zwei Dutzend Professorinnen und Professoren im Departement für Erziehungswissenschaften und mit verschiedenen, relativ stabilen Forschungsteams verfügt sie über eine kritische Masse in der Bildungsforschung. Dazu gehören die einzigen beiden Professuren für "Approches interculturelles de l'éducation" in der Schweiz, eine in "anthropologie de l'éducation et psychologie interculturelle» (PIERRE DASEN - seit 2008 ABDELJALIL AKKARI) und eine weitere in «identités, appartenances, plurilinguisme et pluriculturalité» (CHRISTIANE PERREGAUX - aktuell vakant). Entsprechend findet ein beachtlicher Teil der Bildungsforschung der Schweiz in Genf statt und die «interkulturelle Bildungsforschung» ist in der französischen Schweiz relativ gut verankert.

7 Im weiteren oder engeren Sinne ist dies der Fall an der PH Fachhochschule Nordwestschweiz, PH Bern, PH Bern-Jura-Neuenburg, PH Wallis, PH Schaffhausen, PH Zentralschweiz (und nach wie vor der Universität Genf), an einzelnen Standorten mit besonderer Betonung der Lehrerinnen- und Lehrerbildung (PH Bern, PH Bern-Jura-Neuenburg, PH Zentralschweiz, Universität Genf).

8 Auch bezüglich des Begriffs «interkulturelle Pädagogik» gilt, dass er hier stellvertretend für die vielschichtigen in der Einleitung problematisierten Konzepte verwendet wird.

9 Von insgesamt 17 Pädagogischen Hochschulen und Institutionen der universitären Lehrpersonenbildung.

10 Lehrpersonen, die Studierende während Schulpraktika begleiten.

\section{Literatur}

Allemann-Ghionda, C., de Goumoëns, C. \& Perregaux, C. (1999). Pluralité linguistique et culturelle dans la formation des enseignants. Fribourg: Presses universitaires.

Bade, K. J. \& Bommes, M. (2004). Einleitung. In K. J. Bade \& M. Bommes (Hrsg.), Migration - Integration - Bildung: Grundfragen und Problembereiche. IMIS-Beiträge, Heft 23, Themenheft (S. 7-20). Osnabrück: IMIS.

Becker, R. \& Lauterbach, W. (Hrsg.). (2007). Bildung als Privileg. Theoretische Erklärungen und empirische Befunde zu den Ursachen der Bildungsungleichheiten (2. aktualisierte Aufl.). Wiesbaden: VS-Verlag.

Bennett, C. (2001). Genres of research in multicultural education. Review of Educational Research, 71 (2), 171-217.

Brown, E. L. (2004). What precipitates change in cultural diversity awareness during a multicultural course? The message or the method? Journal of Teacher Education, 55 (4), 325340 .

Cicourel, A. V. \& Kitsuse, J. I. (1974). Die soziale Organisation der Schule und abweichende jugendliche Karrieren. In K. Hurrelmann (Hrsg.), Soziologie der Erziehung (S. 362-378). Weinheim: Beltz.

Cochran-Smith, M., Davis, D. \& Fries, M. K. (2004). Multicultural teacher education research, practice, and policy. In J. A. Banks \& C. McGee Banks (Eds), Handbook of research on multicultural education (pp. 931-975). San Francisco: John Wiley \& Sons.

COHEP (2007). Interkulturelle Pädagogik in der Lehrerinnen- und Lehrerbildung der Schweiz. Grundlagenbericht (Redaktion: Priska Sieber. Verabschiedet von der Mitgliederversammlung COHEP am 14./15. November 2007). Bern: COHEP (Schweizerische Konferenz der Rektorinnen und Rektoren der Pädagogischen Hochschulen).

Criblez, L. (1998). Entre profession et discipline: à propos du statut des sciences de l'éducation en Suisse. In R. Hofstetter \& B. Schneuwly (Ed.) Le pari des sciences de l'éducation. Raisons éducatives, 1/2 (pp. 169-201). Bruxelles: De Boeck. 
EDK (1995). Empfehlungen zur Lehrerbildung und zu den Pädagogischen Hochschulen. Bern: EDK (Schweizerische Konferenz der kantonalen Erziehungsdirektoren).

EDK (1999a). Reglement über die Anerkennung von Hochschuldiplomen für Lehrkräfte der Sekundarstufe I vom 26. August 1999, mit Änderungen vom 28. Oktober 2005. Bern: EDK.

EDK (1999b). Reglement über die Anerkennung von Hochschuldiplomen für Lebrkräfte der Vorschulstufe und der Primarstufe vom 10. Juni 1999, mit Änderungen vom 28. Oktober 2005. Bern: EDK.

EDK/CDIP (2000). Interkulturelle Pädagogik in der Lehrerinnen- und Lehrerbildung. Zentrale Lernbereiche - Thesen - Literaturhinweise. Schlussbericht/Pour une formation des enseignantes et enseignants aux approches interculturelles. Principaux domaines de formation - Propositions pour un curriculum de formation - Indications bibliographiques. Rapport final. Bern: EDK/CDIP (Schweizerische Konferenz der kantonalen Erziehungsdirektoren/Conférence suisse des directeurs cantonaux de l'instruction publique).

Fend, H. (2006). Neue Theorie der Schule: Einführung in das Verstehen von Bildungssystemen (S. 58ff). Wiesbaden: VS Verlag.

Fiechter, U. \& Kappus, E.-N. (2006). Ethnographische Schulforschung. Ungewohnte Perspektive auf schulische Sozialisation. ph akzente, 2, 47-49.

Gakuba, T., Gremion, M., Hutter, V., Ogay, T. \& Zharkova, Y. (en prép.) Bilan de la recherche suisse en éducation interculturelle de 1993 à 2006.

Gogolin, I. (1994). Der monolinguale Habitus der multikulturellen Schule. Münster: Waxmann.

Gogolin, I. (2002). Interkulturelle Bildungsforschung. In R. Tippelt (Hrsg.), Handbuch Bildungsforschung (S. 263-279). Opladen: Leske + Budrich.

Gogolin, I. \& Nauck, B. (Hrsg.). (2000). Migration, gesellschaftliche Differenzierung und Bildung. Opladen: Leske + Budrich.

Gomolla, M. \& Radtke, F.-O. (2002). Institutionelle Diskriminierung. Die Herstellung ethnischer Differenz in der Schule. Opladen: Leske + Budrich.

Gretler, A. (1994). Länderbericht Schweiz. 3. Internationales OECD-Seminar zur Bildungsforschung und -entwicklung. Wien, Oktober 1994. Aarau: SKBF (Schweizerische Koordinationsstelle für Bildungsforschung).

Haeberlin, U., Imdorf, C. \& Kronig, W. (2004). Von der Schule in die Berufslehre. Untersuchungen zur Benachteiligung von ausländischen und von weiblichen Jugendlichen bei der Lehrstellensuche. Bern: Haupt.

Hamburger, F. (1988). Der Kulturkonflikt und seine pädagogische Kompensation. In F. Hamburger (Hrsg.), Pädagogik der Einwanderungsgesellschaft (S. 33-46). Frankfurt a.M.: Cooperative Verlag.

Hofstetter, R. \& Schneuwly, B. (2001). Les sciences de l'éducation en Suisse. Evolution et prospectives, Août 2001. Berne: CEST (Centre d'études de la science et de la technologie).

Kronig, W., Haeberlin, U. \& Eckhart, M. (2000). Immigrantenkinder und schulische Selektion: Pädagogische Visionen, theoretische Erklärungen und empirische Untersuchungen zur Wirkung integrierender und separierender Schulformen in den Grundschuljahren. Bern: Haupt.

Krüger-Potratz, M. (2003). Lehrerbildung im Zeichen von Pluralität und Differenz. In J. Beillerot \& C. Wulf (Hrsg.), Erziehungswissenschaftliche Zeitdiagnosen: Deutschland und Frankreich. Europäische Studien zur Erziehung und Bildung Nr. 20 (S. 83-94). Münster: Waxmann.

Lischer, R. (1997). Integration - (k)eine Erfolgsgeschichte. Ausländische Kinder und Jugendliche im schweizerischen Bildungssystem. Bern: BFS (Bundesamt für Statistik).

Mecheril, P. (2003). Prekäre Verhältnisse. Über natio-ethno-kulturelle (Mehrfach-)Zugehörigkeit (S. $118 \mathrm{ff})$ Münster: Waxmann.

OECD/CERI (2007). National Review of Educational R\&D: Switzerland. Paris: OECD (Organisation for Economic Cooperation and Development) / CERI (Centre for Educational Research and Innovation). 
Oester, K., Fiechter, U. \& Kappus, E.-N. (2005). Schulen in der transnationalen Gesellschaft. Segregations- und Integrationsprozesse am Beispiel Bern West. Forschungsbericht. Bern: Pädagogische Hochschule Bern.

Radtke, F.-O. (2004). Schule und Ethnizität. In W. Helsper \& J. Böhme (Hrsg.), Handbuch der Schulforschung (S. 625-646). Wiesbaden: VS, Verlag für Sozialwissenschaften.

Schütz, A. \& Luckmann, T. (1975). Strukturen der Lebenswelt. Neuwied, Darmstadt: Leuchterhand.

Sieber, P. (2006). Steuerung und Eigendynamik der Aussonderung: Vom Umgang des Bildungswesens mit Heterogenität. Luzern: Edition SZH/CSPS.

Sieber, P. (2007). Der Umgang des Bildungswesens mit migrationsbedingter Vielfalt: historisch gestaltete Institutionen als Rahmen für Ausgrenzungsprozesse. In T. Geisen \& C. Riegel (Hrsg.), Jugend, Partizipation und Migration: Orientierungen im Kontext von Integration und Ausgrenzung (S. 281-304). Wiesbaden: VS-Verlag.

Sieber, P. \& Bischoff, S. (2007). Untersuchung zum Ist-Zustand der Interkulturellen Pädagogik an den Pädagogischen Hochschulen und universitären Lehrerinnen- und Lehrerbildungsinstitutionen der Schweiz (Bericht verabschiedet von der Mitgliederversammlung COHEP am 14./15. November 2007). Bern: COHEP (Schweizerische Konferenz der Rektorinnen und Rektoren der Pädagogischen Hochschulen).

SKBF (2006). Bildungsbericht Schweiz 2006. Aarau: SKBF (Schweizerische Koordinationsstelle für Bildungsforschung).

Sleeter, C. E. (2001). Preparing teachers for culturally diverse schools: Research and the overwhelming presence of whiteness. Journal of Teacher Education, 52 (2), 94-106.

Stuck, O. (2001). Gatekeeping zwischen Individuum, Organisation und Institution. Zur Bedeutung und Analyse von Gatekeeping am Beispiel von Übergängen im Lebenslauf. In L. Leisering, R. Müller \& K. F. Schumann (Hrsg.), Institutionen und Lebensläufe im Wandel: Institutionelle Regulierung von Lebensläufen. Statuspassagen und Lebenslauf, Bd. 2 (S. 2954). Weinheim: Juventa.

Vogel, C. (2006). Forschung und Entwicklung an den Pädagogischen Hochschulen und universitären Lehrerbildungsstätten der Schweiz - Stand und Entwicklungstendenzen 2005: Schlussbericht. Bern: SKPH (Schweizerische Konferenz der Rektorinnen und Rektoren der Pädagogischen Hochschulen).

Schlagworte: Transkulturalität, Pädagogik, Lehrer/innenbildung, Bildungsforschung, Interkulturelle Erziehung 


\title{
Transnationalité et systèmes scolaires nationaux : perspectives pour la recherche et l'enseignement
}

\section{Résumé}

La question posée par ce numéro de la Revue suisse des sciences de l'éducation sur les conséquences, pour la recherche et pour l'enseignement dans la formation des enseignants, du développement des écoles en contextes de vie transnationaux est ici examinée du point de vue particulier de la Suisse alémanique. La thèse avancée est que pour être innovante la recherche doit simultanément considérer l'étude des processus scolaires, appréhender les contextes comme des configurations étatiques nationales et intégrer dans ces réflexions les répercussions possibles sur la pratique scolaire.

En ce qui concerne l'enseignement, nous initions une discussion nuancée à propos des « normes " nationales à l'école et nous soulignons l'importance de recourir à des méthodes orientées vers la pratique. Nous donnons également des indications sur les besoins de développement des Hautes écoles pédagogiques.

Mots clés: Transculturalité, pédagogie, formation des enseignants, recherche en éducation, éducation interculturelle

\section{Transnazionalità e sistemi scolastici nazionali: prospettive per la ricerca e l'insegnamento}

\author{
Riassunto
}

In questo contributo si discutono le questioni relative alle conseguenze degli sviluppi della scuola in contesti di vita transnazionali per la ricerca e l'insegnamento in rapporto alla realtà nella Svizzera tedesca. Si sostiene la tesi che una ricerca innovativa debba indagare processi scolastici, configurare contesti in un'ottica nazionale e discuterne le ricadute per l'insegnamento. Per l'insegnamento si suggerisce un confronto differenziato con le cosiddette "normalità" (nazionali) nelle scuole e si mettono in rilievo i metodi orientati alla pratica. Per concludere l'attenzione verte sulle esigente delle Alte scuole pedagogiche.

Parole chiave: transculturalità, pedagogia, formazione degli insegnanti, ricerca in educazione, educazione interculturale 


\section{Transnationalism and national school systems: Research and teaching perspectives}

\section{Summary}

The development of transnational communities, and their investigation with regards to the requirements of preparing future teachers within teacher training, is addressed in the context of the German speaking area of Switzerland. Research which investigates processes at schools, conceptualizes contexts as state-derived configurations, and reflects upon related effects on educational practices, is considered innovative. Accordingly, the author encourages an in-depth reflection on (national) "normalities" in schools and its strong meaning for practice-oriented methods. Advice is also given with regards to the developmental needs of teacher training institutions.

Key words: Transculturalism, transnationalism, Swiss-German area, teacher education, intercultural education, development of teacher training institutions 